the angles being completely determined by kinematics.

In an experiment reported by D. Schwalm, this simple correlation was used to get rid of the Doppler broadening in a particle- $\gamma$ coincidence experiment. The scattered projectiles were detected in a position sensitive detector, covering a big solid angle. From the scattering angle, the Doppler shift energy was calculated using zero energy loss kinematics which is an excellent approximation because of the high bombarding energies. The Doppler shift itself was calculated relativistically. With that information a corrected spectrum was constructed. The energy resolution is about $3 \mathrm{keV}$ instead of the $50 \mathrm{keV}$ obtained in a direct measurement and this figure can still be improved.

With this refined experimental technique MCE experiments were performed in the actinide region to determine the reduced electrical quadrupole transitions $B(E 2, I \rightarrow I+2)(I=$ even) between successive states. The analysis is straightforward because the excitation mechanism, pure Coulomb interaction, is completely understood. In addition, for a heavy ion experiment, the well known Winther de Boer computer code that calculates the excitation cross sections semi classically, can be used. Thus the $B(E 2)$ values between two successive states can be determined from the intensity of the corresponding line in the spectrum. The number of available $B(E 2)$ values is now pushed to ten or more, which poses a severe test for nuclear models. Although the IB model is quite successful at the lowest levels, there are indications that it fails to explain the trend in the $B(E 2)$ values going up the yrast band. It must be admitted however, that the number of levels excited in experiments so far must be increased by one or two, to have a conclusive result. These improvements are expected in the near future.

\section{Yrast Traps}

A second group of experiments exploiting nuclear fusion processes are used in the search for the so called yrast traps, isomeric states to which the highly excited nucleus decays. The investigated nuclei with mass $A \cong 150$ were produced in a fusion process between a target nucleus of mass $A \cong 110$ and a projectile of mass $A \cong 50$. For a long time yrast traps have been predicted in this mass region. The nucleus formed, leaves the target foil with a high recoil velocity at approx. $0^{\circ}$ and after some $10 \mathrm{~cm}$ of flight is caught by another foil, which is surrounded by a big Nal detector that covers a solid angle of almost $4 \pi$. Through a hole in the $\mathrm{Nal}$ crystal a $\mathrm{Ge}(\mathrm{Li})$ detector observes the catcher foil. During flight all but the isomeric states de-excite to the ground state. Should the isomeric state subsequently decay by a cascade, in the most favourable case, all the $\gamma$ quanta are detected in either the $\mathrm{Ge}(\mathrm{Li})$ or the Nal where a summed peak is received. By triggering the $\mathrm{Ge}(\mathrm{Li})$ with suitable $\mathrm{Nal}$ events, all the members of the cascade and the energy of the isomeric state are yielded. The latter can be taken directly from the $\mathrm{Nal}$, for if the $\mathrm{Ge}(\mathrm{Li})$ spectrum is used, troubles are encountered from the complicated spectrum of competitive transitions. By timing the event against a pulsed beam, the half life of the isomer is also given. By these experiments, the expected high spin isomers have been found in the Gd-region.

Andreas Bockisch

\title{
Quarks and Jets
}

(An abridged version of the original manuscript)

The best way (if not the only way) to understand strong interaction dynamics is by the scattering of particles at high energies. The hope is that in so doing one actually probes the hadronic structure and interacts with the hadronic constituents (i.e. quarks) directly. To illustrate this, we mention the following two very important sets of experiments:-

(i) deep inelestic scattering of leptons off hadrons, and

(ii) "quark jets" and "gluon jets".

(i) In experiments done in 1968/9 at SLAC, quarks/partons were actually "seen" inside the nucleons for the first time. Briefly, by scattering very high-energy electrons off nucleons, the nucleon was shown to consist of two ingredients: an electrically neutral "glue" ( $~ 50 \%$ ) and three point like objects which most probably carry exactly the quark quantum numbers. These are called the "valence quarks". It is also believed that there is a "sea" or "soup" of qव pairs inside the nucleon(s). These observations and late experiments with deep inelastic scattering of neutrinos off nuc- leons (through weak interactions) demonstrated the following two highly significant points:-

(a) at presently available energies, these constituents behave as if they are point-like objects which leads us to the concept of scaling;

(b) at very high energies and large momentum transfers the force between quarks, when they are "very close" to each other is almost zero and the closer they get to each other the freer they become-hence the term: "Asymptotic Freedom". (N.B. Q.CD requires this too).

(ii) The closest one has got so far in trying to observe free quarks is in the production of jets. The first definite indication of "jet structure" was found in 1975 (and confirmed later) in $\mathrm{e}+\mathrm{e}-$ annihilation at the SPEAR storage rings at centre of mass energies above the $\mathrm{J} / \Psi$ resonance region (see Fig. 1).

To search for jets, they first calculated the tensor $\mathrm{Tij}$ for each event: $T^{i j}=\sum_{n}\left(S^{i j} \vec{p}_{n}^{2}-p_{n}^{i} p_{n}^{j}\right)$. i and $j$ refer

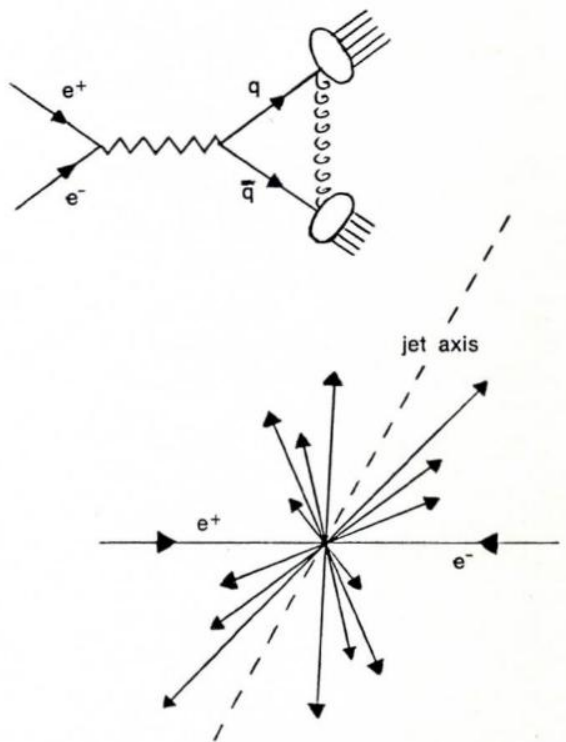

Fig. 1 (Above) Electron-positron annihilation into two quark jets showing the final state interaction;

(below) Example of jets in $e^{+-e^{-}}$annihilation.

to the spatial components of each particle with momentum $\vec{p}_{n}$ and the summation is over all the detected particles $(n=1,2, \ldots)$ in the event. Diagonalizing $\mathrm{T}^{\mathrm{ij}}$ we get the principal moment eigenvalues $\lambda_{1} \lambda_{2}$ and $\lambda_{3}$ in momentum space. Subsequently one defines the sphericity $S$, by $S=$ 
$\lambda_{3} / 1 / 3\left(\lambda_{1},+\lambda_{2}+\lambda_{3}\right)\left(\lambda_{3}\right.$ is assumed to be the smallest eigenvalue). So we see that for jets we should have $S<<1$ (i.e. near zero) and $S \cong 1$ for events with large multiplicity and particles isotropically distributed in phase space.

\section{FREE QUARKS?}

Although from about 1965 physicists have been looking for fractionallycharged single quarks, there is not, as yet, any conclusive evidence as to their existence. At present the search goes on with determination, buoyed up by the fact that at least one of these quarks should be absolutely stable because of charge conservation. Methods mentioned here are not applicable if quarks are unstable and integrally charged (like the ones in the Pati-Salam model). The main searches are being done:- (i) with accelerators, (ii) in cosmic rays, and (iii) in stable matter. The method covered in the Conference was of the third kind and is a "descendent" of the Millikan's oil drop experiment. (see $E N, 1977,7 / 8$, p. 7).

The main improvements in the method since they first started ( 1965) are:-

(i) increasing the mass of the levitated object from

$\mathrm{m} \cong 2 \times 10^{-9} \mathrm{~g}$ to $\mathrm{m} \cong 10^{-4}-2 \times 10^{-4} \mathrm{~g}$ (Cf. Millikan's: $m \cong 10^{-11} \mathrm{~g}$ ) giving thus more chance of observing quarks, and (ii) using the feedback levitation method to keep the test sphere of iron in a fixed horizontal plane.

\section{Strong Interaction Dynamics}

The dynamics of strong interactions has puzzled physicists ever since it was postulated. Due to the very large coupling constant one cannot construct a perturbative theory similar to quantum electrodynamics which agrees so well with experiment, at least at presently available energies. Physicists have invented and used many different techniques in their attempt to understand strong interactions. Among these are dispersion theory, S-Matrix theory, "Regge theory" and Duality. None is adequate to explain all the data

Recently, however the foundations of a quantum field theory (Q.F.T.) of strong interactions have been set, namely "quantum chromodynamics" (Q.CD)

Q.CD is a non-Abelian Q.F.T. of quarks that is locally gauge invariant with respect to (exact) $\mathrm{SU}(3)$ symmetry. The coloured quarks interact via the exchange of coloured vector mesons (i.e. the 8 gluons) corresponding to the 8 generators of $\mathrm{SU}(3)$ colour. Note that in this theory the dynamics depends (or "resides") on colour alone and flavour merely serves as a quark label.

Non-Abelian gauge field theories like Q.CD have some unusual properties. For example:-

(i) the effective interaction between the quarks decreases as the energy and momentum transfer increase. As these variables in- crease asymptotically to infinity, the theory approaches a free field theory.

This property is the Asymptotic Freedom;

(ii) the theory has infrared divergencies. It has been speculated that this property of the theory leads to the confinement of all coloured states, including quarks and gluons. Such confinement is called "infrared slavery"! It is not yet known (conclusively) whether Q.CD does confine quarks and gluons. (A task for the future).

As quarks have not been observed so far, physicists have been trying hard to construct phenomenological models in which quarks are permanently confined. Examples are "the string model(s)" and the Bag models. Bag models have proved very successful, e.g., in explaining the hadronic mass spectrum and the Regge trajectories.

To conclude we compare and contrast Q.ED and Q.CD very briefly:-

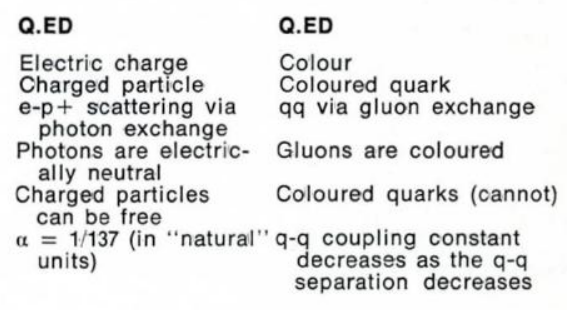

Majid Rahnama

\section{Surfaces and Experimental Techniques}

The extent of current interest in surface physics is indicated by the decision to hold a symposium of five halfdays on the subject, of which one was held in conjunction with the synchrotron radiation group in view of the impact that this new diagnostic source has had. Particular emphasis was placed on the study of semiconductors and surface magnetism.

In the field of semiconductors a great deal of work has been done on relating the electronic properties of the surface and interface to structural characteristics particularly in GaAs. The electronic properties of an ideal heterojunction have been analysed and models of particular systems developed. Imperfections are of great significance in real situations, arising both from intrinsic defects and from impurities present in minute concentrations. Defects have a dramatic ef- fect on the electronic properties of surfaces and on the first stages of oxidation. This is of great importance for example in the preparation of substrates for molecular beam epitaxy, but in recent times it has proved possible to produce monocystalline thin films with precise control of uniformity, thickness and doping profile.

Much has been accomplished in measuring and understanding surface magnetism effects, but in ferromagnetic materials the existence of reduced magnetization near the surface the "dead layer" still remains unexplained.

On the experimental side, the techniques now at the command of the surface physicist are remarkably varied, and provide such a detailed description of surface phenomena it becomes proper to ask how this information can be used. Classical methods include low energy electron diffraction and neutral thermal beam diffraction, the latter providing new information about the gas-surface interaction. With medium energy ion scattering, by using a $100 \mathrm{keV}$ proton beam and measuring the blocking pattern from Rutherford back scatter information is derived on the planar spacing of the first two layers and the location of absorbed atoms. Surface atoms are located with a precision of $0.02 \AA$. High resolution electron loss spectroscopy is particularly well suited to study vibrational spectra of atoms and molecules absorbed on the surfaces of single crystals, and offer a unique possibility of investigating ordered adsorbate structures.

Newly on the scene is infrared photoacoustic spectroscopy whereby an acoustic wave is set up by absorption of a modulated light beam. The me- 